\title{
Proposed Scaling Laws for Laminar Flow of a Bingham Plastic Down an Inclined Plane
}

\author{
C.F. Ihle BRASS Engineering Chile S.A., Chile
}

A. Tamburrino Department of Civil Engineering, Universidad de Chile, Chile

\begin{abstract}
The present tendency of the mining industry to design and evolve their production systems to minimise the use of water has resulted in the need for an assessment of efficient paste and thickened tailings disposal sites. This paper proposes a dimensionless variable and a length model for slow laminar Bingham flows. Results converge to previous scalings at the limit of Newtonian fluid. Reasonable agreement with field data reported in the previous edition of the present conference was found.
\end{abstract}

\section{Introduction}

The way the mining industry views their resource utilisation is changing. A difference from the past is that in many places water is becoming a scarce and expensive resource. Even in places where water supply is not an issue, a sub-optimal use of the resource implies the need of oversized equipment and reservoirs. In particular, lack of water recovery can severely affect tailing deposit dimensions. In South America, several new copper extraction projects include endeavours to minimise the use of water and thus exploit thickened and paste tailings transport and disposal technology. Unlike conventional tailings, thickened and paste tailings technology is not yet mature and there are several features that can still be optimised. In some of the thickened tailing transport project recently developed, for example the Esperanza Project (Antofagasta Minerals S.A., 2008), loop tests have been done to obtain pipeline transport data. Although such an approach is of great use, it may be of little value without a proper physical model to extrapolate the laboratory results to pilot plant parameters. Here, model theory appears as a crucial factor for such a link. On the other hand, a change in the present common-knowledge paradigm that laminar transport is hardly possible is still outstanding (Shou, 2005). New mining projects showing the benefits of proper engineering designs for thickened and paste tailings will certainly change things in the future. This paper deals with one such problem, namely that of thickened and paste tailings flow at the deposit site. Scaling relationships for a simplified idealisation of the real problem that allow a preliminary correlation between laboratory data and design parameters are proposed and tested using previously reported tailings data.

\section{Problem description}

Consider the slow flow of a tailings sheet down the slope of an infinite plane. Tailings are normally deposited from mining plants and correspond to variable-speed viscous gravity currents. Even in the case when discharge is turbulent, they eventually become laminar (Robinsky, 1999). As a first approximation they can be considered as two-dimensional laminar flow. Thus, the flow mainly obeys a buoyancy-viscosity balance different from turbulent Newtonian fluids, the slow gravity currents on slopes (e.g. Benjamin, 1957; Yih, 1963; Lister, 1992) is often viscoplastic. For simplicity, the flow is assumed to be Bingham Plastic. The corresponding stress-strain relationship is given by:

$$
\eta \frac{\partial U}{\partial z}=\left\{\begin{array}{cll}
0 & \text { if } & |\tau|<\tau_{0} \\
\tau_{0} \operatorname{sgn}\left(\frac{\partial U}{\partial z}\right) & \text { if } & |\tau| \geq \tau_{0}
\end{array}\right.
$$

where $U, \eta, \tau$ and $\tau_{0}$ are the horizontal component of velocity, Bingham (dynamic) viscosity, shear and yield stress, respectively. The function sgn is defined as $\operatorname{sgn}(x)=x /|x|$ if $x \neq 0$ and 0 otherwise. Tailings and ore concentrate rheology is due to the presence of highly concentrated particles. The particles, the specific 
gravity of which is commonly much higher than that of water, eventually settle. How fast they settle depends on particle size distribution, solids and liquid density and, importantly, the solids volume concentration. At low particle volume concentrations, settling velocities can be several times higher than those occurring at high concentrations. For volume concentrations $\phi \leq 0.01$ particles-laden flows have been analysed theoretically and experimentally in the light of the lock-release problem (Bonnecaze et al., 1993; Dade and Huppert, 1995; Hallworth et al., 1998), where it is assumed that viscosity enters only in the gravity current runoff length through the Stokes' free fall velocity. In such works, concentration of the solid-liquid mixture is not used in any rheological model, but only in the initial reduced gravity, as $g^{\prime}=\left(\rho_{p}-\rho_{a}\right) \phi_{0}$, where $\phi_{0}, \rho_{p}$, and $\rho_{a}$ are the initial volume concentration, density of the particle and ambient density, respectively. Using much higher concentrations, close to the fluidisation limit, Hallworth and Huppert (1998), show that the above model, based on low-concentration mixtures, is not effective to describe run-off lengths of highly concentrated slurries. They propose a limit of validity around a volume concentration equal to 0.275 . The latter work did not propose a physical explanation for such change in behaviour, which could possibly reside in the nature of rheology, as described in the model by Liu and Mei (1989), based on lubrication theory, where active flow areas co-exist with zones where the stress is below the yield value, as seen from Equation (1). The resulting, so-called plug flow, affects the mean velocity profile and therefore the total flow depth given a volume flow $q_{0}$, affecting the dynamical balance in the system. In two-dimensional flow, the corresponding critical depth, $h p$, corresponds to (Griffiths, 2000):

$$
h_{p}=\frac{\tau_{0}}{\rho_{m} g \sin \theta}
$$

where $g$ is the magnitude of the gravity acceleration vector, and $\rho_{m}$ the density of the pulp. It follows that $\tau=\eta \partial U / \partial z$ for $0 \leq z<H-h_{p} \equiv z^{*}$ and $\tau=0$ for $z^{*} \leq z \leq h$. This implies that for $h=h_{p}$, or $z^{*}=0$, the whole fluid column remains stagnant. This condition is often used to estimate required tailing deposit capacities (Robinsky, 1999). The final condition in Equation (2) does not consider the discharge flow or other properties of the slurry, such as particle size distribution or dynamic viscosity, which also have an effect on the formation of the fluid layer while it flows.

\section{$3 \quad$ Model scaling analysis}

Scales for gravity current height, velocity and length are proposed based on a simplified situation, consisting of a two-dimensional, constant volume flow $q_{0}$ (per unit length) of a thin viscoplastic slurry formed by highly concentrated particles in a liquid substrate flowing on a small slope $\theta$ as shown in Figure 1. It is assumed that the particle size distribution is well represented by a mean particle diameter $\bar{d}$. For practical purposes, the mean particle diameter is assumed to the size corresponding to passing $50 \%$, i.e., $\bar{d}=d_{50}$. Ignoring the influence of settling particles on the dynamics of the flow, fluid motion is well represented by the NavierStokes equation:

$$
\frac{\partial \vec{U}}{\partial t}+\vec{U} \cdot \nabla \vec{U}=-\frac{1}{\rho_{m}} \nabla p+\frac{\eta}{\rho_{m}} \nabla^{2} \vec{U}+\vec{g}
$$

where $\vec{U}=(U, W)^{T}$ is the velocity vector and $p$ the pressure and the coordinate system is depicted in Figure 1. The corresponding boundary conditions are $\tau(z=0)=\rho_{m} g H \sin \theta$ and $\tau(z=H)=0$. Therefore, at some point in the middle there exists a critical height $z^{*}$ where the shear stress is lower than $\tau_{0}$. This point is denoted as $z^{*}=(1-\lambda) H$, with $\lambda<1$. It is noted that when $\lambda \ll<$, the effect of the yield stress is negligible, and the fluid strongly behaves as Newtonian. On the other hand, the case $\lambda \approx 1$ corresponds to the situation when the most of flow is moving as a solid. If $\lambda=1, H=h_{p}$ and the column remains stagnant. Although analytical solutions exist for the two-dimensional unsteady (and consequently) non-uniform flow (Huang and García, 1997), for scaling purposes, the uniform steady state condition gives a good indiction of the dynamic balances within the fluid. Under these assumptions, the velocity field $\vec{U}=U(z)$ corresponding to the solution of Equation (3) is: 


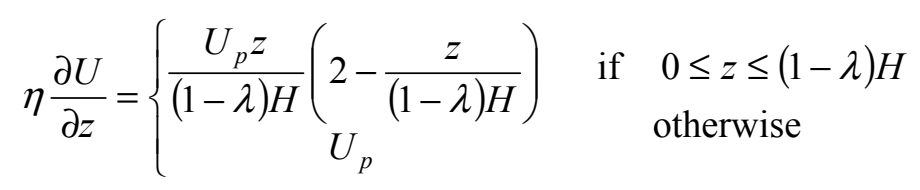

with:

$$
U_{p}=\frac{\rho g(1-\lambda)^{2} H^{2} \sin \theta}{2 \eta}
$$

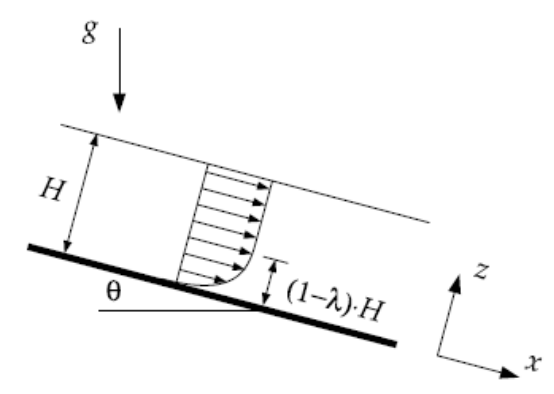

\section{Figure 1 Schematic of the problem}

The corresponding average velocity is given by

$$
\bar{U}=\frac{a \tau_{w} H}{\eta}\left[1-b \frac{\tau_{0}}{\tau_{w}}+c\left(\frac{\tau_{0}}{\tau_{w}}\right)^{3}\right],
$$

where $\tau_{w}=\rho_{m} g H \sin \theta,(a, b, c)=(1 / 3,3 / 2,1 / 2)$ is the shear stress at the bottom of the flow. This relation is similar to that found in other works. For instance, modifying the Buckingham equation (Wasp, 1977), neglecting the fourth order term in the Buckingham equation and using a hydraulic radius for the case of an open channel with finite walls, Abulnaga (2002) model yields $(a, b, c)=(1 / 2,4 / 3,0)$. Other models for finitewidth channels are described on Alderman and Haldenwang (2007).

The parameter $\lambda$ could be also written as the ratio between the fluid depth and the depth given by Equation (2):

$$
\lambda=\frac{\tau_{0}}{H \rho_{m} g \sin \theta} .
$$

Assuming the inflow $q_{0}$ is known, an integral volume conservation statement can be imposed:

$$
q_{0}=\int_{0}^{H} U\left(z^{\prime}\right) d z^{\prime} .
$$

Combining Equation (7) and Equation (8) yields:

$$
N-(h-1)^{2}(2 h+1)=0,
$$

where $h=H \rho_{m} g \sin \theta / \tau_{0}$ and:

$$
N=\frac{6 \eta q_{0}\left(\rho_{m} g \sin \theta\right)^{2}}{\tau_{0}^{3}}
$$

is a control parameter. Equation (9) has only one real solution if $N(N-1)>0$ (Abramowitz and Stegun, 1972). 
Given fluid properties and flow, $N$ is always positive. If $N>1$, the non-dimensional flow depth is given by:

$$
h=\frac{1}{2^{2 / 3}}\left\{\left[N+N^{1 / 2}(N-1)^{1 / 2}-\frac{1}{2}\right]^{1 / 3}+\left[N-N^{1 / 2}(N-1)^{1 / 2}-\frac{1}{2}\right]^{1 / 3}+\frac{1}{2^{1 / 3}}\right\} .
$$

A plot of the solution is shown in Figure 2. It is noted that for large values of $N, h \sim(N / 2)^{1 / 3}$ or, in dimensional terms,

$$
H_{N \gg 1} \sim\left(\frac{3 \eta q_{0}}{\rho_{m} g \sin \theta}\right)^{1 / 3}
$$

which is independent of the yield stress and, moreover, corresponds to the asymptotic solution for large values of time for the low-Reynolds-number Newtonian flow found by Lister (1992). Equation (11) may be considered as a length scale of a thin Bingham sheet flow. It is meaningful as long as flow is laminar. If the flow is turbulent, $\tau \gg>\tau_{0}$ and the effect of viscosity becomes unimportant. In this case, the buoyancy is no longer balanced by viscosity, but rather by inertial forces, given by $\partial \vec{U} / \partial t+\vec{U} \cdot \nabla \vec{U}$.

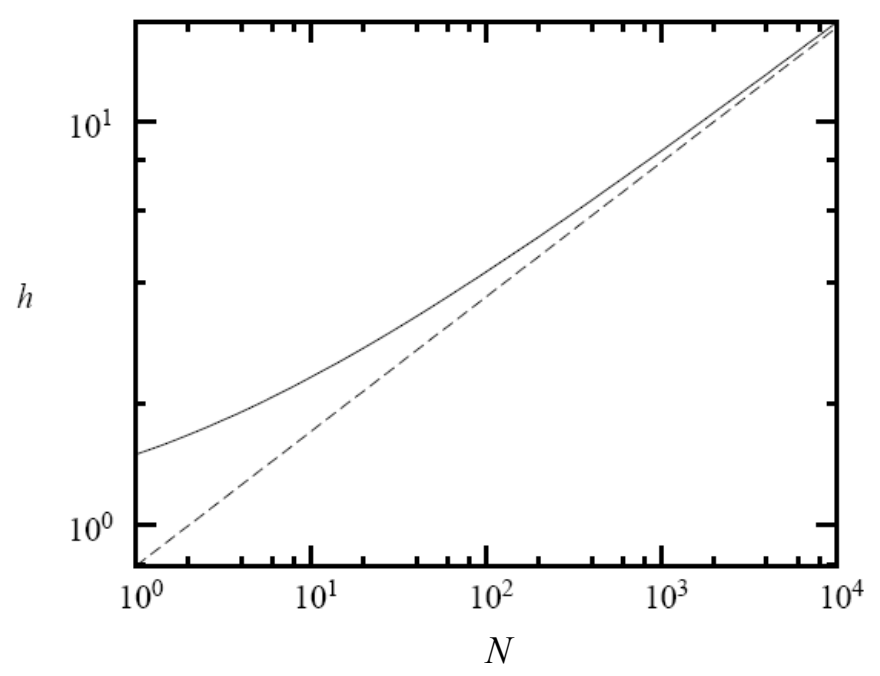

Figure 2 Plot of the nondimensional flow depth $h$ in terms of the dimensionless parameter $N$. The dashed line corresponds to $(\mathrm{N} / 2)^{1 / 3}$

A velocity relationship can be defined from Equation (11) and the flow $q_{0}$ as:

$$
\widetilde{U}=\frac{q_{0}}{H}=\frac{q_{0} \rho_{m} g \sin \theta}{h \tau_{0}} .
$$

A time relationship for the advance of the front can be obtained out the flow depth scale and that of the velocity:

$$
t_{\widetilde{U}}=\frac{h^{2} \tau_{0}^{2}}{q_{0}\left(\rho_{m} g \sin \theta\right)^{2}} .
$$

Equation (14) also corresponds to that obtained using the time scale corresponding to the strain rate, scaled as $\dot{\gamma}^{-1}=H / \widetilde{U}$. The horizontal displacement of the slurry sheet in laminar flow features the sedimentation of particles. If the concentration of solid particles in volume, $\phi$, is large enough, particles interact among themselves through the carrying fluid to lower down the mean sedimentation velocity. This phenomenon, called hindered settling, has been studied in the case of mudflows. 
The following semi-empirical relation has been widely used to compute the hindered settling velocity $W_{\phi}$ (Richardson and Zaki, 1954):

$$
\frac{W_{\phi}}{W_{\infty}}=(1-\phi)^{n}
$$

where $W_{\infty}=d_{p}^{2}\left(\rho_{s}-\rho_{l}\right) g /\left(18 \eta_{l}\right)$ is the Stokes free-fall velocity of a single particle of diameter $d_{p}$ in an infinite medium, with $\rho_{s}$ the solid density, $\rho_{l}$ and $\eta_{l}$ the density and dynamic viscosity of the liquid. The exponent $n$ has been found to be about 4.5 for fine-medium sands (Baldock et al., 2004, and references therein) and close to 5 for finer particles. A time scale for the sedimentation process, corresponding to the mean time it takes a particle to settle, can be estimated as $t_{s}=H / W_{\phi}$. Whenever $t_{s}$ is large compared to $t_{\widetilde{U}}$, sedimentation is not likely to induce a significant change on flow properties and therefore the relationships proposed in this paper are valid.

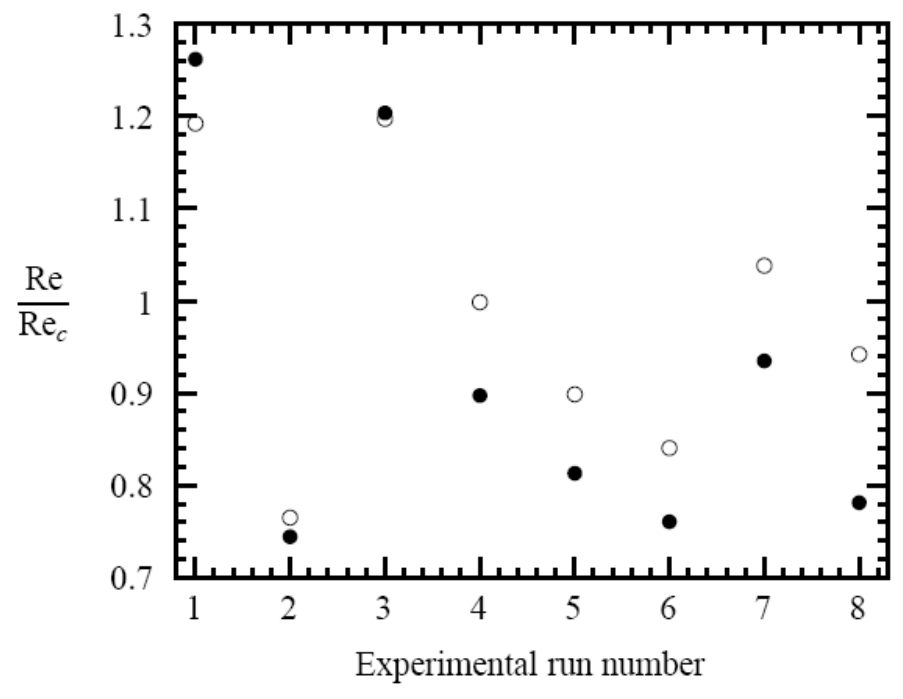

Figure $3 R e / R e c$ ratio as a function of the Hedström number for Pirouz et al. (2005) data. Circles represent critical values obtained with Naik (1983) transition model, while solid circles represent the Hanks (1963) data

\section{$4 \quad$ Results and discussion}

The scaling relationship that yields the nondimensional number $N$ and the flow depth scale $h$, as shown in Equation (11), is confirmed against field experimental data using gold tailings (Pirouz et al., 2005). As the latter flows were confined in semi-circular channels of different diameters, the hydraulic radius was considered as a equivalent length parameter. The latter authors point out that according to the flow Reynolds numbers they computed and the velocity profiles the flow is turbulent. However, they did not assess the effect of the yield stress on their computation of the flow transition. Defining the Reynolds and Hedström numbers as:

$$
R e=\frac{4 \rho_{m} U R_{h}}{\eta} ; \quad H e=\frac{16 \rho R_{h}^{2} \tau_{0}}{\eta^{2}}
$$

based on pipeline flow, Hanks (1963) and Naik (1983) propose critical Reynolds numbers given the Hedström number as defined in Equation (16) as:

$$
R e_{c}=\frac{H e}{a}\left(1-b r_{\tau}+c r_{\tau}^{3}\right)
$$


where $r_{\tau}$ is bottom stress to critical shear stress ratio, and is such that:

$$
\frac{r_{\tau}}{\left(1-r_{\tau}\right)^{3}}=\frac{H e}{d}
$$

The parameters $a, b, c$ and $d$ correspond to $8,4 / 3,1 / 3$ and 16800 in Hanks (1963) and 12, 3/2, 1/2 and 48000 in Naik (1983), respectively. In the light of Pirouz et al. (2005) and both models, some of the corresponding Reynolds numbers appear to be subcritical, as depicted in Figure 3. On the other hand, for the same data set, the time scale ratio of horizontal flow to that corresponding to particle sedimentation is shown. This is depicted on Figure 4, where values between $10^{-4}$ and $10^{-5}$ were found. Therefore, for this laminar and transitional regime, sedimentation is not expected to significantly modify rheology and, consequently, $N$.

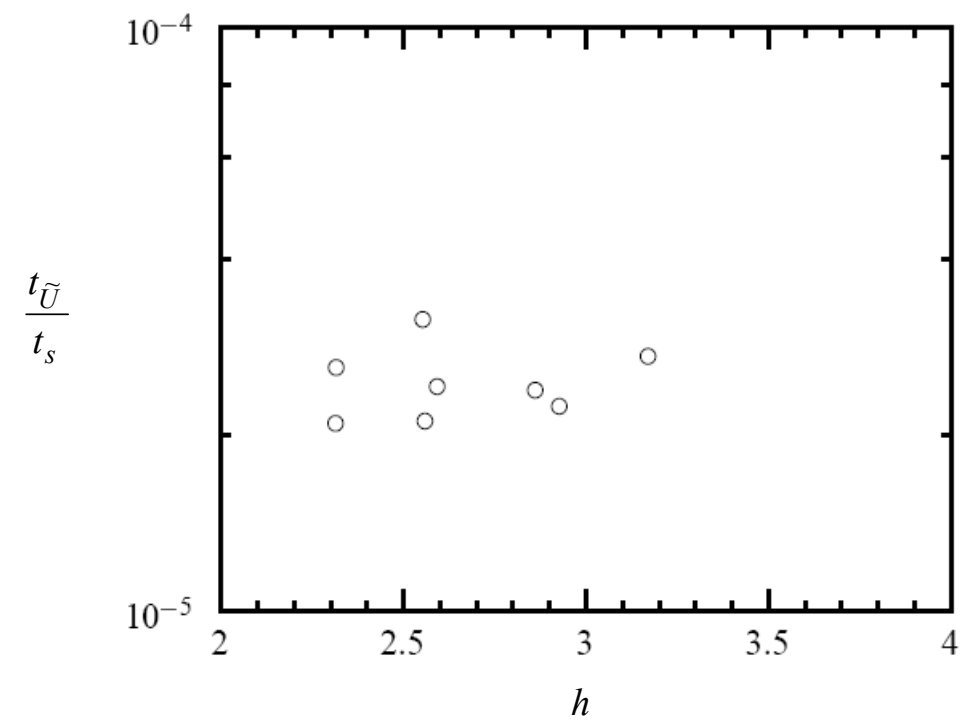

Figure 4 Time scale ratio ${ }^{t} / t_{s}$ using the hydraulic radius for Pirouz et al. (2005) data, in terms of the dimensionless fluid depth as defined in Equation (11)

Flow depths, scaled as hydraulic radius from Pirouz et al. (2005) are compared with the scaling for $h$ corresponding to Equation (11). Differences between $15 \%$ and $30 \%$ are observed from experimental data, as shown in Figure 5. The largest $r$, correspond to the highest Reynolds number flow. Part of this difference is explained by the transitional nature of the flow. On the other hand, one of the main assumptions of the deduction of Equation (11) is the existence of a two-dimensional flow or, similarly, by not considering the wall effects on the channel. In the case of a tailing disposal site, this is a plausible assumption. Confined, semicircular channels do inevitably yield additional friction, and therefore larger flow depths than in the present, idealised model.

Friction losses can be estimated theoretically from the present model out of the definition $f=2 \tau_{w} /\left(\tau_{m} U^{2}\right)$. In terms of $h, N$ and $\widetilde{U}, f=\Pi h^{3} / N$, with $\Pi=12 \eta /\left(\rho_{m} q_{0}\right)$. In the limit of Newtonian flow, that is, $N \rightarrow \infty$, it follows that $f \sim R e^{-1}$. Although experimental data from Pirouz et al. (2005) is promising in this sense, a broader $N$ and parameter range is required to validate present scalings, ensuring the flow is fully in the laminar range. Such a set of experimental test work is presently done by our research group, with special attention on the effect of the yield stress, which is very important when dealing with highly concentrated slurries.

\section{Conclusions}

The study of thickened tailings deposition poses a challenge for engineers today. In contrast to conventional tailings, thickened and paste Bingham Plastics present difficulties for the assessment of tailing disposal sites. Although simple tools do currently exist, proper knowledge of high concentration sheet flows is still lacking. 
Although a usual practice for the design of disposal sites is to do pilot tests, differences between laboratory and field test data is fairly common, and most likely explained by the lack of use of proper models that are able to model, and scalem the most important dynamic balances occurring in the flow. This has motivated the present work where, defining a new dimensionless variable and length relationship derived from the solution of a steady and uniform solution of a Bingham Plastic, has been proposed to account for the relative effects of viscoplastic and sheared flow behaviour. Results are compared to reported data in the literature with fair agreement. At the limit of Newtonian behaviour, the present proposed scaling relationship converges to a previously proposed, and experimentally validated, theoretical model. Further laminar, twodimensional experiments are presently being done to be able to find the proportionality factor for the length scaling law and, importantly, a scaling relationship for the Fanning friction factor.

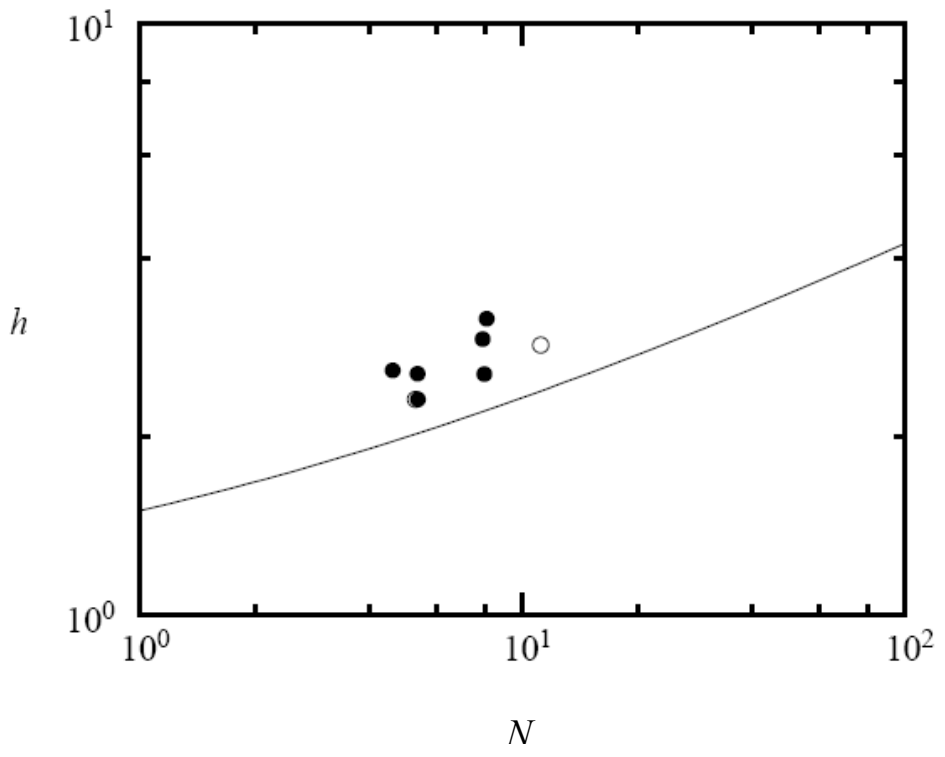

Figure 5 Solid curve: computed flow height $h$, obtained from Equation (11). Circles: tailings hydraulic radius by Pirouz et al. (2005), non-dimensionalised by $\tau 0(\rho m g \sin \theta)-1$. Hollow circles represent the condition $\operatorname{Re} / \operatorname{Rec}<0.9$, while solid ones, $\operatorname{Re} / \operatorname{Rec} \geq 0.9$

\section{Acknowledgements}

The authors would like to thank Mr Luis Navarro for useful discussions regarding the preparation of the present work.

\section{References}

Abramowitz, M. and Stegun, I.A. (eds) (1972) Handbook of mathematical functions, US National Bureau of Standards, 10th edition.

Abulnaga, B.E. (2002) Slurry sistems handbook, McGraw-Hill.

Alderman, N.J. and Haldenwang, R. (2007) A review of Newtonian and non-Newtonian flow in rectangular open channels, In Hydrotransport 17, 17th International Conference on the Hydraulic Transport of Solids.

Antofagasta Minerals S.A. Esperanza project overview (2008) URL http://www.mineraesperanza.cl, viewed 14th October 2008.

Baldock, T.E., Tomkins, M.R., Nielsen, P. and Hughes, M.G. (2004) Settling velocity of sediments at high concentrations, Coast. Eng., 51, pp. 91-100.

Benjamin, T.B. (1957) Wave formation in a laminar flow down an inclined plate. J. Fluid Mech., 2, pp. 554-574.

Bonnecaze, R.T., Huppert, H.E. and Lister, J.R. (1993) Particle-driven gravity currents. J. Fluid Mech., 250, pp. 339-369.

Dade, W.B. and Huppert, H.E. (1995) Runout and fine-sediment deposits of axisymmetric turbidity currents, J. Geophys. Res., 100(C9), pp. 18,597-18, 609.

Griffiths, R.W. (2000) The dynamics of lava flows. Annu. Rev. Fluid Mech., 32, pp. 477-518.

Hallworth, M.A. and Huppert, H.E. (1998) Abrupt transitions in high-concentration, particle-driven gravity currents, Phys. Fluids, 10(5), pp. 1083-1087. 
Hallworth, M.A., Hogg, A.J. and Huppert, H.E. (1998) Effects of external flow on compositional and particle gravity currents, J. Fluid Mech., 359, pp.109-142.

Hanks, R.W. (1963) The laminar-turbulent transition in pipes, concentric annuli and parallel plates, AIChE J., 9, pp. $173-181$.

Huang, X. and García, M.H. (1997) A perturbation solution for Bingham-plastic mudflows, J. Hidr. Eng., 123, pp. 986-994.

Lister, J.R. (1992) Viscous flows down an inclined plane from point and line sources, J. Fluid Mech., 242 , pp. 631-653.

Liu, K.O. and Mei, C.C. (1989) Slow spreading of a sheet of bingham fluid on an inclined plane, J. Fluid Mech., 207, pp. 505-529.

Naik, B. (1983) Mechanics of mudflow and treated as the flow of a Bingham fluid, PhD thesis, Washington State University.

Pirouz, B., Kavianpour, M.R. and Williams, P. (2005) Thickened tailings beach deposition, field observations and fullscale flume testing, Proceedings of the Eighth International Seminar on Paste and Thickened Tailings, R.J. Jewell and S. Barrera (eds), Australian Centre for Geomechanics, Perth, Australia, pp. 53-72.

Richardson, J. F. and Zaki, W.N. (1954) Sedimentation and fluidisation; part 1, Trans. Inst. Chem. Eng., 32, pp. 35-53.

Robinsky, E.I. (1999) Thickened tailings disposal in the mining industry, E.I. Robinsky Associates Limited.

Shou, G. (2005) Laminar flow - possible pipeline operating mode, Proceedings of the Eighth International Seminar on Paste and Thickened Tailings, R.J. Jewell and S. Barrera (eds), Australian Centre for Geomechanics, Perth, Australia, pp. 45-52.

Wasp, E.J. (1977) Solid-Liquid Flow Slurry Pipeline Transportation, Trans Tech Publications.

Yih, C.S. (1963) Stability of liquid flow down an inclined plane. Phys. Fluids, 6, pp. 321-334. 